\title{
Continuous dependence and convergence for a Kelvin-Voigt fluid of order one
}

\author{
Brian Straughan ${ }^{1}$
}

Received: 19 April 2021 / Accepted: 1 November 2021 / Published online: 22 November 2021

(c) The Author(s) 2021

\begin{abstract}
It is shown that the solution to the boundary - initial value problem for a KelvinVoigt fluid of order one depends continuously upon the Kelvin-Voigt parameters, the viscosity, and the viscoelastic coefficients. Convergence of a solution is also shown.
\end{abstract}

Keywords Continuous dependence - Kelvin-Voigt - A priori bound · Viscoelasticity

\section{Introduction}

The equations for a viscoelastic fluid have been increasingly occupying attention. Such fluids occur everywhere in real life and differ from Navier - Stokes fluids in that the stress depends on the history of the velocity gradient. As such, the equations for such fluids present many mathematical challenges, see e.g. [1-15].

A particular class of viscoelastic fluids of interest here are those associated with the names of Kelvin and of Voigt, see e.g. [16-21]. Much of the interest in these fluids stems from work of Russian writers in this field and analytical studies of Kelvin-Voigt fluids are contained in $[22,23]$, with generalizations of these models and analytical results to encompass the non - isothermal situation in [24-26]. A lucid account of viscoelastic fluids associated with the names of Maxwell, of Oldroyd, and of Kelvin and Voigt, is contained in [27], where the solution existence question is analysed, see also [28].

Kelvin-Voigt fluids are being increasingly employed in real life applications especially in industrial and engineering contexts. Many of these are reviewed in [19], but we highlight here their employment in viscous dampers in large buildings, cf. [29,30]. For example, a large viscous damper is utilized in the 1667 feet high tower Taipei 101 in the city of Taipei. This building has been constructed to withstand earthquakes and typhoons and the large viscous damper is essential.

Brian Straughan

brian.straughan@durham.ac.uk

1 Department of Mathematics, University of Durham, Durham DH1 3LE, UK 
The goal of this article is to analyse continuous dependence of a solution to the equations for a Kelvin-Voigt fluid of order one. Such questions are important and belong to the general area of structural stability. [31], p. 304, pose the problem of what effect does changing the parameters in a differential equation have upon the solution to such an equation. They introduce this as the concept of structural stability. In this article we concentrate on continuous dependence on parameters in the equations for a Kelvin-Voigt fluid of order one. This is continuous dependence on the model itself which is structural stability of the model. We point out that continuous dependence on the model has been the subject of much recent attention in continuum mechanics, see e.g. $[13,16,32-54]$.

In the next section we introduce the Kelvin-Voigt equations of order one. The following section establishes continuous dependence on the Kelvin-Voigt parameter, $\lambda$. This is important as this coefficient multiplies the highest derivative term in the equations. After this we establish continuous dependence upon the remaining coefficients in the governing equations.

\section{The Kelvin-Voigt equations of order one}

Throughout this article we employ standard indicial notation in conjunction with the Einstein summation convention. Hence, partial differentiation with respect to $x_{i}$ is written as ${ }_{, i} \equiv \partial / \partial x_{i}$, and $\Delta$ denotes the Laplacian in $\mathbb{R}^{3}$.

Let $v_{i}(\mathbf{x}, t)$ be the velocity, $p(\mathbf{x}, t)$ be the pressure, and let $f_{i}(\mathbf{x}, t)$ be the body force at position $\mathbf{x}$ and time $t$. Now, let $q_{i}^{m}(\mathbf{x}, t), m=1, \ldots, L$, be viscoelastic variables. Then [27] define a hierarchy of viscoelastic fluid models. They write that a Maxwell fluid of order $L, L \in \mathbb{N}$, satisfies the equations

$$
\begin{aligned}
& v_{i, t}+v_{j} v_{i, j}-\sum_{m=1}^{L} \beta_{m}^{(1)} \Delta q_{i}^{m}+p_{, i}=f_{i}, \\
& v_{i, i}=0 \\
& q_{i, t}^{m}+\gamma_{m} q_{i}^{m}=v_{i}, \quad m=1, \ldots, L .
\end{aligned}
$$

An Oldroyd fluid of order $L, L \in \mathbb{N}$, satisfies the equations

$$
\begin{aligned}
& v_{i, t}+v_{j} v_{i, j}-\mu^{(2)} \Delta v_{i}-\sum_{m=1}^{L} \beta_{m}^{(2)} \Delta q_{i}^{m}+p_{, i}=f_{i}, \\
& v_{i, i}=0, \\
& q_{i, t}^{m}+\gamma_{m} q_{i}^{m}=v_{i}, \quad m=1, \ldots, L .
\end{aligned}
$$

A Kelvin-Voigt fluid of order $L, L \in \mathbb{N}$, satisfies the equations

$$
v_{i, t}+v_{j} v_{i, j}-\lambda \Delta v_{i, t}-\mu^{(3)} \Delta v_{i}-\sum_{m=1}^{L} \beta_{m}^{(3)} \Delta q_{i}^{m}+p_{, i}=f_{i},
$$




$$
\begin{aligned}
& v_{i, i}=0, \\
& q_{i, t}^{m}+\gamma_{m} q_{i}^{m}=v_{i}, \quad m=1, \ldots, L .
\end{aligned}
$$

In equations (1) - (3), the coefficients $\beta_{m}^{(k)}, k=1,2,3, m=1, \ldots, L, \gamma_{m}, m=$ $1, \ldots, L, \mu^{(2)}, \mu^{(3)}$ and $\lambda$ are positive constants, and it should be noted that there is no sum on $m$ in the terms $\gamma_{m} q_{i}^{m}$.

The nonlinearity in equations (1) - (3) consists of the $v_{j} v_{i, j}$ term. An equation which has been studied is one for the so called Navier - Stokes - Voigt fluid, cf. the very interesting results on attractors and regularity by [55], [56]. The Navier - Stokes - Voigt equations, which are also known as the Kelvin-Voigt equations of order zero, are

$$
\begin{aligned}
& v_{i, t}+v_{j} v_{i, j}-\lambda \Delta v_{i, t}-\mu \Delta v_{i}+p_{, i}=f_{i}, \\
& v_{i, i}=0 .
\end{aligned}
$$

These equations also contain the nonlinear term $v_{j} v_{i, j}$ and a very interesting article establishing the existence of weak solutions and of strong solutions in appropriate $L^{q}(\Omega)$ spaces for $1<q<\infty$ with $\Omega$ a bounded domain in $\mathbb{R}^{d}, d \geq 2$, is due to [57]. These writers raise the question of how equations (4) might be derived from the balance of linear momentum and continuity equations for a viscous incompressible fluid, namely,

$$
\begin{aligned}
& v_{i, t}+v_{j} v_{i, j}=T_{j i, j}+f_{i}, \\
& v_{i, i}=0,
\end{aligned}
$$

where $T_{i j}$ is the symmetric Cauchy stress tensor. They argue that some writers present for equations (4) a constitutive equation of form

$$
T_{i j}=-p \delta_{i j}+2 \mu d_{i j}+2 \lambda d_{i j, t},
$$

where $d_{i j}=\left(v_{i, j}+v_{j, i}\right) / 2$, and they point out that this is not correct since the tensor $d_{i j, t}$ is not objective. Thus, the term $-\lambda \Delta v_{i, t}$ in (4) should be regarded as a regularization term for the Navier - Stokes equations.

One may use the [57] argument also for equations (3). However, a fully nonlinear model for a Navier - Stokes - Voigt fluid is derived by [58]. This model is now referred to as a Walters fluid, see e.g. [59]. The model of [58] recognizes the fact that $d_{i j, t}$ is not objective as [57] point out and they replace it with an objective derivative of form

$$
d_{i j}^{\nabla}=d_{i j, t}+v_{k} d_{i j, k}-v_{j, k} d_{i k}-v_{i, k} d_{k j}
$$


see also [60], [59]. This requires one to analyse instead of (4) the momentum equation

$$
\begin{aligned}
v_{i, t}+v_{j} v_{i, j}= & -p_{, i}+\mu \Delta v_{i}+\lambda \Delta v_{i, t} \\
& +\lambda\left[v_{k}\left(v_{i, j}+v_{j, i}\right)_{, k}\right]_{, j} \\
& -\lambda\left[v_{j, k}\left(v_{i, k}+v_{k, i}\right)\right]_{, j} \\
& -\lambda\left[v_{i, k}\left(v_{k, j}+v_{j, k}\right)\right]_{, j} .
\end{aligned}
$$

Here the divergence of $\mathbf{v}$ is also zero. If we employ this argument for a Kelvin-Voigt fluid of order one then instead of equation (3) 1 with $L=1$ we should use (7) with a $-\beta \Delta q_{i}$ term added to the right hand side.

Of particular interest to the present article are equations (1) - (3) for fluids of order one, i.e. when $L=1$. [13] study continuous dependence and convergence for a linear system arising from the Maxwell equations (1) and we develop linear equations here. In the linear case we write the analogous systems to (1) - (3) of order one as, with $f_{i}=0$,

Maxwell,

$$
\begin{aligned}
& v_{i, t}-\beta \Delta q_{i}+p_{, i}=0, \\
& v_{i, i}=0, \\
& q_{i, t}+\gamma q_{i}=v_{i},
\end{aligned}
$$

Oldroyd,

$$
\begin{aligned}
& v_{i, t}-\mu \Delta v_{i}-\beta \Delta q_{i}+p_{, i}=0, \\
& v_{i, i}=0, \\
& q_{i, t}+\gamma q_{i}=v_{i},
\end{aligned}
$$

Kelvin-Voigt,

$$
\begin{aligned}
& v_{i, t}-\lambda \Delta v_{i, t}-\mu \Delta v_{i}-\beta \Delta q_{i}+p_{, i}=0, \\
& v_{i, i}=0, \\
& q_{i, t}+\gamma q_{i}=v_{i} .
\end{aligned}
$$

It is sometimes convenient to eliminate $q_{i}$ in these equations and derive the following equations for $v_{i}$ and a generalized pressure, of form,

Maxwell,

$$
\begin{aligned}
& v_{i, t t}-\beta \Delta v_{i}+\gamma v_{i, t}=-\phi_{, i}, \\
& v_{i, i}=0,
\end{aligned}
$$

Oldroyd,

$$
\begin{aligned}
& v_{i, t t}-\mu \Delta v_{i, t}+\gamma v_{i, t}-(\beta+\mu \gamma) \Delta v_{i}=-\phi_{, i}, \\
& v_{i, i}=0,
\end{aligned}
$$


Kelvin-Voigt,

$$
\begin{aligned}
& v_{i, t t}-\lambda \Delta v_{i, t t}-(\gamma \lambda+\mu) \Delta v_{i, t}+\gamma v_{i, t}-(\beta+\mu \gamma) \Delta v_{i}=-\phi_{, i}, \\
& v_{i, i}=0,
\end{aligned}
$$

where $\phi=p_{, t}+\gamma p$.

We observe that formally, as $\lambda \rightarrow 0$ the Kelvin-Voigt system (13) tends to the Oldroyd equations (12). As $\mu \rightarrow 0$ the Oldroyd equations (12) tend to the Maxwell equations (11). Furthermore, if we put $\gamma=\beta=1 / \zeta$ and rescale the pressure $\phi$ as $\phi=\psi / \zeta$, then the Maxwell system (11) becomes

$$
\begin{aligned}
& \zeta v_{i, t t}-\Delta v_{i}+v_{i, t}=-\psi_{, i}, \\
& v_{i, i}=0
\end{aligned}
$$

When $\zeta \rightarrow 0$, equations (14) tend to those of Stokes flow, cf. [13].

In this article we concentrate on equations (10) or (13) and we establish continuous dependence of the solution upon the parameters $\lambda, \beta, \gamma$ and $\mu$.

\section{Continuous dependence upon the Kelvin-Voigt coefficient $\lambda$}

As remarked at the end of the introduction, the Kelvin-Voigt coefficient multiplies the highest derivative term in (10) or (13), and as such it is highly important that the solution depends continuously on changes in this parameter. To establish continuous dependence upon $\lambda$ we let $\left(u_{i}, q_{i}^{1}, p^{1}\right)$ and $\left(v_{i}, q_{i}^{2}, p^{2}\right)$ be solutions to equations (10) for fixed constants $\mu, \beta$ and $\gamma$, but for $\lambda_{1}$ and $\lambda_{2}$, respectively. In each case the equations are defined on a bounded region $\Omega$ in $\mathbb{R}^{3}$ with boundary $\Gamma$ which is sufficiently regular to allow application of the divergence theorem. We here restrict attention to a bounded domain in $\mathbb{R}^{3}$ although the methods work for a bounded domain in $\mathbb{R}^{n}, n \geq 2$.

Thus, $\left(u_{i}, q_{i}^{1}, p^{1}\right)$ satisfies the boundary - initial value problem

$$
\begin{aligned}
& u_{i, t}-\lambda_{1} \Delta u_{i, t}-\mu \Delta u_{i}-\beta \Delta q_{i}^{1}=-p_{, i}^{1}, \\
& u_{i, i}=0, \\
& q_{i, t}^{1}+\gamma q_{i}^{1}=u_{i},
\end{aligned}
$$

on $\Omega \times(0, T)$, for some $T>0$, with the boundary and initial conditions,

$$
\begin{aligned}
& u_{i}=\ell_{i}(\mathbf{x}), \quad \mathbf{x} \in \Gamma, \\
& u_{i}(\mathbf{x}, 0)=f_{i}(\mathbf{x}), \quad q_{i}^{1}(\mathbf{x}, 0)=h_{i}(\mathbf{x}), \quad \mathbf{x} \in \Omega,
\end{aligned}
$$

for prescribed functions $f_{i}, h_{i}$ and $\ell_{i}$. In general, one should like $\ell_{i}(\mathbf{x})$ to also depend on $t$. The method we employ requires us to allow $\ell_{i}$ to depend only on $\mathbf{x}$, which was also the case in [13]. 
The solution $\left(v_{i}, q_{i}^{2}, p^{2}\right)$ satisfies the boundary - initial value problem

$$
\begin{aligned}
& v_{i, t}-\lambda_{2} \Delta v_{i, t}-\mu \Delta v_{i}-\beta \Delta q_{i}^{2}=-p_{, i}^{2}, \\
& v_{i, i}=0 \\
& q_{i, t}^{2}+\gamma q_{i}^{2}=v_{i},
\end{aligned}
$$

on $\Omega \times(0, T)$, for some $T>0$, with the boundary and initial conditions,

$$
\begin{aligned}
& v_{i}=\ell_{i}(\mathbf{x}), \quad \mathbf{x} \in \Gamma, \\
& v_{i}(\mathbf{x}, 0)=f_{i}(\mathbf{x}), \quad q_{i}^{2}(\mathbf{x}, 0)=h_{i}(\mathbf{x}), \quad \mathbf{x} \in \Omega .
\end{aligned}
$$

Define the difference variables $w_{i}, q_{i}, \pi$ and $\lambda$ as

$$
w_{i}=u_{i}-v_{i}, \quad q_{i}=q_{i}^{1}-q_{i}^{2}, \quad \pi=p^{1}-p^{2}, \quad \lambda=\lambda_{1}-\lambda_{2} .
$$

Then $\left(w_{i}, q_{i}, \pi\right)$ satsifies the boundary - initial value problem

$$
\begin{aligned}
& w_{i, t}-\lambda \Delta u_{i, t}-\lambda_{2} \Delta w_{i, t}-\mu \Delta w_{i}-\beta \Delta q_{i}=-\pi_{, i}, \\
& w_{i, i}=0, \\
& q_{i, t}+\gamma q_{i}=w_{i},
\end{aligned}
$$

on $\Omega \times(0, T)$, for some $T>0$, with the boundary and initial conditions,

$$
\begin{aligned}
& w_{i}=0, \quad \mathbf{x} \in \Gamma, \\
& w_{i}(\mathbf{x}, 0)=0, \quad q_{i}(\mathbf{x}, 0)=0, \quad \mathbf{x} \in \Omega .
\end{aligned}
$$

Let now $(\cdot, \cdot)$ and $\|\cdot\|$ denote the inner product and norm on $L^{2}(\Omega)$.

We commence the continuous dependence analysis by multiplying (19) $)_{1}$ by $w_{i}$ and integrating over $\Omega$. After use of the boundary conditions one obtains

$$
\frac{d}{d t}\left(\frac{1}{2}\|\mathbf{w}\|^{2}+\frac{\lambda}{2}\|\nabla \mathbf{w}\|^{2}\right)+\mu\|\nabla \mathbf{w}\|^{2}=-\lambda\left(u_{i, t j}, w_{i, j}\right)-\beta\left(w_{i, j}, q_{i, j}\right) .
$$

Next we differentiate equation (19) 3 with respect to $x_{j}$ and $t$, multiply by $q_{i, j}$ and integrate over $\Omega$ to find

$$
\frac{d}{d t} \frac{1}{2}\|\nabla \mathbf{q}\|^{2}+\gamma\|\nabla \mathbf{q}\|^{2}=\left(w_{i, j}, q_{i, j}\right) .
$$

Upon forming $(21)+\beta \times(22)$ one derives

$$
\begin{gathered}
\frac{d}{d t}\left(\frac{1}{2}\|\mathbf{w}\|^{2}+\frac{\lambda_{2}}{2}\|\nabla \mathbf{w}\|^{2}+\frac{\beta}{2}\|\nabla \mathbf{q}\|^{2}\right)+\beta \lambda\|\nabla \mathbf{q}\|^{2}+\mu\|\nabla \mathbf{w}\|^{2} \\
=-\lambda\left(u_{i, t j}, w_{i, j}\right)
\end{gathered}
$$




$$
\leq \frac{\lambda^{2}}{2 \epsilon}\left\|\nabla \mathbf{u}_{, t}\right\|^{2}+\frac{\epsilon}{2}\|\nabla \mathbf{w}\|^{2}
$$

for $\epsilon>0$, where we have employed the arithmetic - geometric mean inequality. Pick $\epsilon=\mu$ and integrate over $(0, t)$ to find

$$
\begin{gathered}
\frac{1}{2}\|\mathbf{w}\|^{2}+\frac{\lambda_{2}}{2}\|\nabla \mathbf{w}\|^{2}+\frac{\beta}{2}\|\nabla \mathbf{q}\|^{2}+\beta \gamma \int_{0}^{t}\|\nabla \mathbf{q}\|^{2} d s \\
+\frac{\mu}{2} \int_{0}^{t}\|\nabla \mathbf{w}\|^{2} d s \leq \frac{\lambda^{2}}{2 \mu} \int_{0}^{t}\left\|\nabla \mathbf{u}_{, s}\right\|^{2} d s .
\end{gathered}
$$

We now proceed to bound the right hand side of (24) in terms of data. To do this we differentiate $(15)_{1,3}$ to obtain

$$
\begin{aligned}
& u_{i, t t}-\lambda_{1} \Delta u_{i, t t}-\mu \Delta u_{i, t}-\beta \Delta q_{i, t}^{1}=-p_{, i t}^{1}, \\
& q_{i, t t j}^{1}+\gamma q_{i, t j}^{1}=u_{i, t j} .
\end{aligned}
$$

To employ $(25)_{1}$ we additionally prescribe $u_{i, t}(\mathbf{x}, 0)=g_{i}(\mathbf{x})$. Multiply $(25)_{1}$ by $u_{i, t}$ and integrate over $\Omega$ noting $u_{i, t}=0$ on $\Gamma$. Multiply (25) 2 by $q_{i, t j}^{1}$ and integrate over $\Omega$. After some manipulation one may obtain

$$
\frac{d}{d t}\left(\frac{1}{2}\left\|\mathbf{u}_{, t}\right\|^{2}+\frac{\lambda_{1}}{2}\left\|\nabla \mathbf{u}_{, t}\right\|^{2}+\frac{\beta}{2}\left\|\nabla \mathbf{q}_{, t}^{1}\right\|^{2}\right)+\mu\left\|\nabla \mathbf{u}_{, t}\right\|^{2}+\beta \gamma\left\|\nabla \mathbf{q}_{, t}^{1}\right\|^{2}=0 .
$$

This equation is integrated over $(0, t)$ to find using the initial data

$$
\begin{aligned}
& \frac{1}{2}\left\|\mathbf{u}_{, t}\right\|^{2}+\frac{\lambda_{1}}{2}\left\|\nabla \mathbf{u}_{, t}\right\|^{2}+\frac{\beta}{2}\left\|\nabla \mathbf{q}_{, t}^{1}\right\|^{2}+\mu \int_{0}^{t}\left\|\nabla \mathbf{u}_{, s}\right\|^{2} d s \\
& \quad+\beta \gamma \int_{0}^{t}\left\|\nabla \mathbf{q}_{, s}^{1}\right\|^{2} d s=\frac{1}{2}\|\mathbf{g}\|^{2}+\frac{\lambda_{1}}{2}\|\nabla \mathbf{g}\|^{2}+\|\nabla \mathbf{f}-\gamma \nabla \mathbf{g}\|^{2} \\
& \quad=d_{0},
\end{aligned}
$$

where $d_{0}$ is the data term defined in (26). From this expression one deduces

$$
\int_{0}^{t}\left\|\nabla \mathbf{u}_{, s}\right\|^{2} d s \leq \frac{1}{\mu} d_{0} .
$$

Employ this in (24) to obtain the inequality

$$
\begin{aligned}
\frac{1}{2}\|\mathbf{w}\|^{2} & +\frac{\lambda_{2}}{2}\|\nabla \mathbf{w}\|^{2}+\frac{\beta}{2}\|\nabla \mathbf{q}\|^{2}+\beta \gamma \int_{0}^{t}\|\nabla \mathbf{q}\|^{2} d s \\
+\frac{\mu}{2} \int_{0}^{t}\|\nabla \mathbf{w}\|^{2} d s & \leq \frac{d_{0}}{2 \mu^{2}} \lambda^{2} .
\end{aligned}
$$

Inequality (27) establishes continuous dependence of a solution on $\lambda$ in the measures $\|\mathbf{w}\|,\|\nabla \mathbf{w}\|$, and $\|\nabla \mathbf{q}\|$. 
Remark If we set $\lambda_{2}=0$ and put $\lambda_{1}=\lambda$ then (27) yields a convergence result indicating how $u_{i}$ converges to $v_{i}$, a solution to the Oldroyd order one equations.

\section{Continuous dependence upon the parameters $\mu, \beta, \gamma$}

Let now $\left(u_{i}, q_{i}^{1}, p^{1}\right)$ and $\left(v_{i}, q_{i}^{2}, p^{2}\right)$ be solutions to the Kelvin-Voigt order one equations (10) for the same coefficient $\lambda$, but with values $\mu_{1}, \beta_{1}, \gamma_{1}$, and $\mu_{2}, \beta_{2}, \gamma_{2}$, respectively. Thus, these solutions satisfy the boundary - initial value problems

$$
\begin{aligned}
& u_{i, t}-\lambda \Delta u_{i, t}-\mu_{1} \Delta u_{i}-\beta_{1} \Delta q_{i}^{1}=-p_{, i}^{1}, \\
& u_{i, i}=0, \\
& q_{i, t}^{1}+\gamma_{1} q_{i}^{1}=u_{i},
\end{aligned}
$$

on $\Omega \times(0, T)$, with

$$
\begin{aligned}
& u_{i}=\ell_{i}(\mathbf{x}), \quad \mathbf{x} \in \Gamma, \\
& u_{i}(\mathbf{x}, 0)=f_{i}(\mathbf{x}), \quad q_{i}^{1}(\mathbf{x}, 0)=h_{i}(\mathbf{x}), \quad \mathbf{x} \in \Omega,
\end{aligned}
$$

and

$$
\begin{aligned}
& v_{i, t}-\lambda \Delta v_{i, t}-\mu_{2} \Delta v_{i}-\beta_{2} \Delta q_{i}^{2}=-p_{, i}^{2}, \\
& v_{i, i}=0, \\
& q_{i, t}^{2}+\gamma_{2} q_{i}^{2}=v_{i},
\end{aligned}
$$

on $\Omega \times(0, T)$, with

$$
\begin{aligned}
& v_{i}=\ell_{i}(\mathbf{x}), \quad \mathbf{x} \in \Gamma, \\
& v_{i}(\mathbf{x}, 0)=f_{i}(\mathbf{x}), \quad q_{i}^{2}(\mathbf{x}, 0)=h_{i}(\mathbf{x}), \quad \mathbf{x} \in \Omega .
\end{aligned}
$$

Define the difference variables by

$$
\begin{aligned}
& w_{i}=u_{i}-v_{i}, \quad q_{i}=q_{i}^{1}-q_{i}^{2}, \quad \pi=p^{1}-p^{2}, \\
& \mu=\mu_{1}-\mu_{2}, \quad \beta=\beta_{1}-\beta_{2} \quad \gamma=\gamma_{1}-\gamma_{2} . .
\end{aligned}
$$

One then finds that the difference solution satisfies the boundary - initial value problem

$$
\begin{aligned}
& w_{i, t}-\lambda \Delta w_{i, t}-\mu \Delta u_{i}-\mu_{2} \Delta w_{i}-\beta \Delta q_{i}^{1}-\beta_{2} \Delta q_{i}=-\pi_{, i}, \\
& w_{i, i}=0, \\
& q_{i, t}+\gamma q_{i}^{1}+\gamma_{2} q_{i}=w_{i}
\end{aligned}
$$


on $\Omega \times(0, T)$, with

$$
\begin{aligned}
& w_{i}=0, \quad \mathbf{x} \in \Gamma, \\
& w_{i}(\mathbf{x}, 0)=0, \quad q_{i}(\mathbf{x}, 0)=0, \quad \mathbf{x} \in \Omega .
\end{aligned}
$$

Next, differentiate (32) 3 with respect to $x_{j}$, and multiply the result by $q_{i, j}$ and integrate over $\Omega$. Multiply (32) $)_{1}$ by $w_{i}$ and integrate over $\Omega$. After some integration by parts and use of the boundary conditions one may combine the results to derive

$$
\begin{aligned}
\frac{d}{d t}\left(\frac{1}{2}\|\mathbf{w}\|^{2}\right. & \left.+\frac{\lambda}{2}\|\nabla \mathbf{w}\|^{2}+\frac{\beta_{2}}{2}\|\nabla \mathbf{q}\|^{2}\right)+\mu_{2}\|\nabla \mathbf{w}\|^{2}+\gamma_{2} \beta_{2}\|\nabla \mathbf{q}\|^{2} \\
& =-\mu\left(u_{i, j}, w_{i, j}\right)-\beta\left(q_{i, j}^{1}, w_{i, j}\right)-\beta_{2} \gamma\left(q_{i, j}^{1}, q_{i, j}\right) .
\end{aligned}
$$

We next employ the arithmetic - geometric mean inequality on the right hand side of this equation to see that

$$
\begin{aligned}
\frac{d}{d t}\left(\frac{1}{2}\|\mathbf{w}\|^{2}\right. & \left.+\frac{\lambda}{2}\|\nabla \mathbf{w}\|^{2}+\frac{\beta_{2}}{2}\|\nabla \mathbf{q}\|^{2}\right)+\mu_{2}\|\nabla \mathbf{w}\|^{2}+\gamma_{2} \beta_{2}\|\nabla \mathbf{q}\|^{2} \\
& \leq \frac{2}{\mu_{2}} \mu^{2}\|\nabla \mathbf{u}\|^{2}+\left(\frac{2}{\mu_{2}} \beta^{2}+\frac{1}{\beta_{2} \gamma_{2}} \gamma^{2}\right)\left\|\nabla \mathbf{q}^{1}\right\|^{2} .
\end{aligned}
$$

We need to bound the right hand side of (34) in terms of $\mu^{2}, \beta^{2}$ and $\gamma^{2}$. To do this we observe that $u_{i}$ satsify the equations (13) for $\gamma_{1}, \mu_{1}, \beta_{1}$, and we impose $u_{i, t}(\mathbf{x}, 0)=$ $g_{i}(\mathbf{x})$. We multiply the appropriate version of $(13)_{1}$ by $u_{i, t}$ and integrate over $\Omega$ to obtain after integration by parts and integration over $(0, t)$,

$$
\begin{aligned}
\frac{1}{2}\left\|\mathbf{u}_{, t}\right\|^{2} & +\frac{\lambda}{2}\left\|\nabla \mathbf{u}_{, t}\right\|^{2}+\left(\frac{\beta_{1}+\gamma_{1} \mu_{1}}{2}\right)\|\nabla \mathbf{u}\|^{2} \\
& +\left(\gamma_{1} \lambda+\mu_{1}\right) \int_{0}^{t}\left\|\nabla \mathbf{u}_{, s}\right\|^{2} d s+\gamma_{1} \int_{0}^{t}\left\|\mathbf{u}_{, s}\right\|^{2} d s \\
& =\frac{1}{2}\|\mathbf{g}\|^{2}+\frac{\lambda}{2}\|\nabla \mathbf{q}\|^{2}+\left(\frac{\beta_{1}+\gamma_{1} \mu_{1}}{2}\right)\|\nabla \mathbf{f}\|^{2} .
\end{aligned}
$$

Furthermore, differentiate $(28)_{3}$ to find

$$
q_{i, j t}^{1}+\gamma_{1} q_{i, j}^{1}=u_{i, j}
$$

Multiply this by $q_{i, j}^{1}$ and integrate over $\Omega$ to find after use of the arithmetic - geometric mean inequality

$$
\frac{d}{d t}\left\|\nabla \mathbf{q}^{1}\right\|^{2}+\gamma_{1}\left\|\nabla \mathbf{q}^{1}\right\|^{2} \leq \frac{1}{\gamma_{1}}\|\nabla \mathbf{u}\|^{2} .
$$

Let $d_{1}$ be the data term

$$
d_{1}=\frac{1}{\left(\beta_{1}+\gamma_{1} \mu_{1}\right)}\|\mathbf{g}\|^{2}+\frac{\lambda}{\left(\beta_{1}+\gamma_{1} \mu_{1}\right)}\|\nabla \mathbf{g}\|^{2}+\|\nabla \mathbf{f}\|^{2} .
$$


Then from (35) we deduce

$$
\|\nabla \mathbf{u}\|^{2} \leq d_{1}
$$

We employ this estimate in (36) and integrate to obtain

$$
\left\|\nabla \mathbf{q}^{1}\right\|^{2} \leq \frac{1}{\gamma_{1}^{2}} d_{1}+\|\nabla \mathbf{g}\|^{2} e^{-\gamma_{1} t}=d_{2}
$$

where $d_{2}$ is the indicated data term. Upon employment of (37) and (38) in (34) and a further integration we may obtain

$$
\begin{aligned}
\frac{1}{2}\|\mathbf{w}\|^{2} & +\frac{\lambda}{2}\|\nabla \mathbf{w}\|^{2}+\frac{\beta_{2}}{2}\|\nabla \mathbf{q}\|^{2} \\
& +\frac{\mu_{2}}{2} \int_{0}^{t}\|\nabla \mathbf{w}\|^{2} d s+\frac{\gamma_{2} \beta_{2}}{2} \int_{0}^{t}\|\nabla \mathbf{q}\|^{2} d s \leq d_{3},
\end{aligned}
$$

where $d_{3}$ is the data term (involving $\mu^{2}, \beta^{2}, \gamma^{2}$ )

$$
d_{3}=\frac{2 d_{1} t}{\mu_{2}} \mu^{2}+\left(\frac{2}{\mu_{2}} \beta^{2}+\frac{1}{\beta_{2} \gamma_{2}} \gamma^{2}\right)\left(\frac{d_{1} t}{\gamma_{1}^{2}}+\frac{1}{\gamma_{1}}\|\nabla \mathbf{g}\|^{2}\right) \text {. }
$$

Inequality (39) demonstrates continuous dependence of the solution upon the parameters $\mu, \beta$ and $\gamma$.

Acknowledgements I am indebted to two anonymous referees for very helpful comments which led to substantial improvements, and for pointing out some important references.

\section{Compliance with ethical standards}

Conflicts of interest There are no conflicts of interest.

Open Access This article is licensed under a Creative Commons Attribution 4.0 International License, which permits use, sharing, adaptation, distribution and reproduction in any medium or format, as long as you give appropriate credit to the original author(s) and the source, provide a link to the Creative Commons licence, and indicate if changes were made. The images or other third party material in this article are included in the article's Creative Commons licence, unless indicated otherwise in a credit line to the material. If material is not included in the article's Creative Commons licence and your intended use is not permitted by statutory regulation or exceeds the permitted use, you will need to obtain permission directly from the copyright holder. To view a copy of this licence, visit http://creativecommons.org/licenses/by/4.0/.

\section{References}

1. Amendola, G., Fabrizio, M.: Thermal convection in a simple fluid with fading memory. J. Math. Anal. Appl. 366, 444-459 (2010)

2. Amendola, G., Fabrizio, M., Golden, M., Lazzari, B.: Free energies and asymptotic behaviour for incompressible viscoelastic fluids. Applicable Anal. 88, 789-805 (2009) 
3. Anand, V., David, J.R.J., Christov, I.C.: Non-Newtonian fluid structure interactions: static response of a microchannel due to internal flow of a power law fluid. Int. J. Non Newtonian Fluid Mech. 264, 67-72 (2019)

4. Anand, V., Christov, I.C.: Transient compressible flow in a compliant viscoelastic tube. Phys. Fluids 32, 112014 (2020)

5. Anand, V., Christov, I.C.: Revisiting steady viscous flow of a generalized Newtonian fluid through a slender elastic tube using shell theory. Z. Angew. Math. Mech. 101, e201900309 (2021)

6. Christov, I.C., Christov, C.I.: Stress retardation versus stress relaxation in linear viscoelasticity. Mech. Res. Comm. 72, 59-63 (2016)

7. Coscia, V., Sequeira, A., Videman, J.: Existence and uniqueness of classical solutions for a class of complexity - 2 fluids. Int. J. Nonlinear Mech. 68, 531-551 (2015)

8. Fabrizio, M., Lazzari, B., Nibbi, R.: Aymptotic stability in linear viscoelasticity with supplies. J. Math. Anal. Appl. 427, 629-645 (2015)

9. Franchi, F., Lazzari, B., Nibbi, R.: Mathematical models for the non - isothermal Johnson - Segalman viscoelasticity in porous media: stability and wave propagation. Math. Meth. Appl. Sci. 38, 4075-4087 (2015a)

10. Franchi, F., Lazzari, B., Nibbi, R.: The Johnson - Segalman model versus a non - ideal MHD theory. Phys. Lett. A 379, 1431-1436 (2015b)

11. Jordan, P.M., Puri, A., Boros, G.: On a new exact solution to Stokes' first problem for Maxwell fluids. Int. J. Nonlinear Mech. 39, 1371-1377 (2004)

12. Jordan, P.M., Puri, A.: Revisiting Stokes' first problem for Maxwell fluids. Q. J1. Mech. Appl. Math. 58, 213-227 (2005)

13. Payne, L.E., Straughan, B.: Convergence for the equations of a Maxwell fluid. Stud. Appl. Math. 103, 267-278 (1999a)

14. Vuk, E.: Un teorema di esistenza e unicitá per un fluido viscoso con memoria. Annali dell'Universitá di Ferrara 36, 63-72 (1990)

15. Yang, R., Christov, I.C., Griffiths, I.M., Ramon, G.Z.: Time - averaged transport in oscillatory flow of a viscoelastic fluid. Phys. Rev. Fluids 5, 094501 (2020)

16. Chirita, S., Zampoli, V.: On the forward and backward in time problems in the Kelvin - Voigt thermoelastic materials. Mech. Res. Comm. 68, 25-30 (2015)

17. Chirita, S., Ciarletta, M., Tibullo, V.: Rayleigh surface waves on a Kelvin - Voigt viscoelastic half space. J. Elasticity 115, 61-76 (2014)

18. Rivera, J.M., Racke, R.: Transmission problems in (thermo) viscoelasticity with Kelvin - Voigt damping: non-exponential, strong and polynomial stability. SIAM J. Math. Anal. 49, 3741-3765 (2017)

19. Straughan, B.: Instability thresholds for thermal convection in a Kelvin-Voigt fluid of variable order. Circ. Matem. Palermo, Rend (2021). https://doi.org/10.1007/s12215-020-00588-1

20. Svanadze, M.M.: On the solutions of equations of linear thermoelastic theory for Kelvin-Voigt materials with voids. J. Thermal Stresses 12, 253-269 (2014)

21. Svanadze, M.M.: External boundary value problems in the quasi static theory of thermoviscoelasticity for Kelvin-Voigt materials with double porosity. Proc. Appl. Math. Mech. 17, 469-470 (2017)

22. Oskolkov, A.P.: Initial-boundary value problems for the equations of Kelvin - Voigt fluids and Oldroyd fluids. Proc. Steklov Inst. Math. 179, 126-164 (1988)

23. Oskolkov, A.P.: Nonlocal problems for the equations of motion of Kelvin-Voigt fluids. J. Math. Sciences 75, 2058-2078 (1995)

24. Sukacheva, T.G., Matveeva, O.P.: On a homogeneous model of the non-compressible viscoelastic Kelvin-Voigt fluid of the non-zero order. J. Samara State Tech. Univ. Ser. Phys. Math. Sci. 5, 33-41 (2010)

25. Matveeva, O.P.: Model of thermoconvection of incompressible viscoelastic fluid of non - zero order computational experiment. Bull. South Ural State Tech. Univ., Ser. Math. Modelling and Programming, 6:134-138, (2013)

26. Sukacheva, T.G., Kondyukov, A.O.: On a class of Sobolev type equations. Bull. South Ural State Tech. Univ., Ser. Math. Modelling and Programming, 7:5-21, (2014)

27. Oskolkov, A.P., Shadiev, R.: Towards a theory of global solvability on $[0, \infty)$ of initial-boundary value problems for the equations of motion of Oldroyd and Kelvin - Voigt fluids. J. Math. Sci. 68, 240-253 (1994)

28. Christov, I.C., Jordan, P.M.: Maxwell's "other" equations. The Royal Society, Blog (2015). (https:// royalsociety.org/blog/2015/09/maxwells-other-equations) 
29. Greco, R., Marano, G.C.: Identification of parameters of Maxwell and Kelvin-Voigt generalized models for fluid viscous dampers. J. Vibration Control 21, 260-274 (2015)

30. Lewandowski, R., Chorazyczewski, B.: Identification of the parameters of the Kelvin-Voigt and the Maxwell models, used to modelling viscoelastic dampers. Comput. Struct. 88, 1-17 (2010)

31. Hirsch, M.W., Smale, S.: Differential equations, dynamical systems, and linear algebra. Academic Press (1974)

32. Celebi, O.A., Kalantarov, V.K.: Decay of solutions and structural stability for the coupled KuramotoSivashinsky-Ginzburg-Landau equations. Appl. Anal. 94, 2342-2354 (2015)

33. Celik, E., Hoang, L.: Maximum estimates for generalized Forchheimer flows in heterogeneous porous media. J. Diff. Eq. 262, 2158-2195 (2017)

34. Chirita, S.: Well - posed problems. In R. B. Hetnarski, editor, Encyclopedia of thermal stresses. Springer, Dordrecht, (2014). https://doi.org/10.1007/978-94-007-2739-7_264

35. Chirita, S.: On the time differential dual-phase-lag thermoelasticity model. Meccanica 52, 349-361 (2017)

36. Chirita, S.: Modelling triple porosity under local thermal nonequilibrium. J. Thermal Stresses. (2020). https://doi.org/10.1080/01495739.2019.1679057

37. Chirita, S., D'Apice, C., Zampoli, V.: The time differential three-phase-lag heat conduction model: thermodynamic compatibility and continuous dependence. Int. J. Heat Mass Transfer 102, 226-232 (2016)

38. Christov, I.C., Jordan, P.M.: On an instability exhibited by the ballistic-diffusive heat conduction model of Xu and Hu. Proc. Roy. Soc. London A 470, 20130557 (2014)

39. Carasso, A.: Logarithmic convexity and the "slow evolution" constraint in ill posed initial value problems. SIAM J. Math. Anal. 30, 479-496 (1999)

40. Carasso, A.: Reconstructing the past from imprecise knowledge of the present: some examples of non uniqueness in solving parabolic equations backward in time. Math. Meth. Appl. Sci. 36, 249-261 (2013)

41. Franchi, F., Nibbi, R., Straughan, B.: Continuous dependence on modelling for temperature dependent bidispersive flow. Proc. Roy. Soc. London A 473, 20170485 (2017)

42. Ciarletta, M., Straughan, B., Tibullo, V.: Structural stability for a thermal convection model with temperature - dependent solubility. Nonlinear Anal. Real World Appl. 22, 34-43 (2015)

43. Gentile, M., Straughan, B.: Structural stability in resonant penetrative convection in a Forchheimer porous material. Nonlinear Anal. Real World Appl. 14, 397-401 (2013)

44. Guo, B., Guo, C.: The convergence of non-Newtonian fluids to Navier-Stokes equations. J. Math. Anal. Appl. 357, 468-478 (2009)

45. Hoang, L., Ibragimov, A.: Structural stability of generalized Forchheimer equations for compressible fluids in porous media. Nonlinearity 24, 1-41 (2011)

46. Jordan, P.M., Dai, W., Mickens, R.E.: A note on the delayed heat equation; instability with respect to initial data. Mech. Res. Comm. 35, 414-420 (2008)

47. Kutev, N., Kolkovska, N., Dimova, M.: Nonexistence of global solutions to new ordinary differential inequality and applications to nonlinear dispersive equations. Math. Meth. Appl. Sci. 39, 2287-2297 (2016)

48. Liu, Y.: Continuous dependence for a thermal convection model with temperature-dependent solubility. Appl. Math. Comp. 308, 18-30 (2017)

49. Liu, Y., Xiao, S., Lin, Y.W.: Continuous dependence for the Brinkman-Forchheimer fluid interfacing with a Darcy fluid in a bounded domain. Math. Comp. Simulation 150, 66-88 (2018)

50. Liu, Y., Xiao, S.: Structural stability for the Brinkman fluid interfacing with a Darcy fluid in an unbounded domain. Nonlinear Anal. Real World Appl. 42, 308-333 (2018)

51. Li, Y., Zhang, S., Lin, C.: Structural stability for the Brinkman equations interfacing with Darcy equations in a bounded domain. Value Prob, Bound (2021). https://doi.org/10.1186/s13661-021-015010

52. Payne, L.E., Straughan, B.: Stability in the initial - time geometry problem for the Brinkman and Darcy equations of flow in porous media. J. Math. Pures Appl. 75, 225-271 (1996)

53. Payne, L.E., Straughan, B.: Analysis of the boundary condition at the interface between a viscous fluid and a porous medium and related modelling questions. J. Math. Pures Appl. 77, 317-354 (1998)

54. Payne, L.E., Straughan, B.: Effect of errors in the spatial geometry for temperature dependent Stokes flow. J. Math. Pures Appl. 78, 609-632 (1999b) 
55. Kalantarov, V.K., Titi, E.S.: Global attractors and determining modes for the 3D Navier-Stokes-Voigt equations. Chinese Annals of Math. 30, 697-714 (2009)

56. Kalantarov, V.K., Levant, B., Titi, E.S.: Gevrey regularity of the global attractor of the 3D NavierStokes-Voigt equations. J. Nonlinear Sci. 19, 133-152 (2009)

57. Damázio, P.D., Manholi, P., Silvestre, A.L.: $L^{q}$ - theory of the Kelvin-Voigt equations in bounded domains. J. Diff. Eq. 260, 8242-8260 (2016)

58. Beard, D.W., Walters, K.: Elastic-viscous boundary layer flows I Two-dimensional flow near a stagnation point. Math. Proc. Cambridge Philos. Soc. 60, 667-674 (1964)

59. Palese, L.: Thermosolutal convection in a rotating viscoelastic Walters fluid. Atti Accad. Peloritana Pericolanti, Cl. Sci. Fis., Matem., Nat., 77:A20, (2019)

60. Burdujan, I.: The flow of a particular class of Oldroyd - B fluids. Annals Acad, Romanian Scientists: Series Math. Applic., 3:23-45, (2011). Issue in memory of Adelina Georgescu

Publisher's Note Springer Nature remains neutral with regard to jurisdictional claims in published maps and institutional affiliations. 